\title{
DISPOSITIFS NUMÉRIQUES ET EXPÉRIENTIALITÉ
}

\author{
Stéphanie Le MAÎTRE ${ }^{1}$
}

Le journalisme narratif, loin des codes contemporains de l'information, n'est plus aujourd'hui l'apanage du papier : le web est devenu un autre terrain d'expression de ce type de récit médiatique. Grâce aux outils qui s'y déploient les journalistes trouvent d'autres moyens de raconter leurs histoires et de créer une expérience par procuration pour leurs lecteurs. Cet article se propose d'interroger les stratégies déployées par les journalistes narratifs en termes d'expérientialité à l'aune des dispositifs numériques. Il s'agit ici de s'intéresser à la manière dont les professionnels construisent leurs récits sur le web et mettent à profit les différents formats numériques pour créer ou renforcer l'expérientialité du lecteur face au récit qui lui est donné à lire sur son écran.

La frontière entre journalisme et littérature a toujours été extrêmement ténue. Les journaux ouvrant régulièrement leurs colonnes aux hommes de lettres dès le $\mathrm{XIX}^{\mathrm{e}}$ siècle, tandis que les journalistes piochent fréquemment dans la boîte à outils des écrivains pour construire leurs articles. Il est un type de journalisme qui revendique cette proximité, cette intimité pourrait-on dire, avec la littérature : le journalisme narratif. Celui-ci « évolue dans un "entre-deux-narratif", dans lequel l'impératif réaliste de l'éthique journalistique n'entrave pas la célébration du style et la recherche du suspens via des procédés de mise en tension nar-

1 Stéphanie Le Maître est doctorante en Sciences de l'Information et de la Communication au sein du laboratoire GERiiCO (Université de Lille).

Recherches en communication, $\mathrm{n}^{\circ} 51$ - Article publié le 10/09/2020 
rative » (Pélissier, 2012). En effet, le journalisme narratif repose largement sur les techniques de narration habituellement réservées aux écrivains pour rendre compte du réel. Il s'appuie sur un « arc narratif [...] dont les composant[s] princip[aux] sont les protagonistes, la complication, une tension narrative, un dénouement »(Lallemand, 2011). Cet arc narratif met en scène des personnages dont la vie intérieure est exposée, en s'appuyant sur des détails concrets, afin de parvenir à créer une expérience pour le lecteur (Pélissier, 2012 ; Vanoost, 2013).

Cette dimension expérientielle fait écho au concept formulé pour la première fois par Monika Fludernik (1996) sous le terme d'« expérientialité ». Entendu comme l'essence même de la narrativité, le terme désigne la manière dont un récit permet l'investissement psychologique du lecteur. Celui-ci appréhendera alors les personnages et les situations du récit de la même manière que les expériences de la vie réelle (Fludernik, 1996 ; Caracciolo, 2013). Prise dans le cadre du journalisme, cette expérientialité doit servir la compréhension des questions d'actualité, puisque « le récit reste le meilleur lieu de médiation pour l'appropriation des événements du monde par les citoyens consommateurs d'information » (Lits, 2007).

Il semble que le journalisme narratif soit remis sur le devant de la scène médiatique francophone depuis quelques années, notamment par l'intermédiaire du « mook», contraction de magazine et de book. Ces revues prennent le temps médiatique à rebours et proposent, à la fois du point de vue de l'immersion et du compte rendu réalisé, de longues enquêtes journalistiques mises en scène au moyen des « ressources de la fiction » (Pélissier \& Eyriès, 2016). La revue XXI constitue l'exemple le « plus frappant » de ce choix à contre-courant (Vanoost, 2013), et il apparaît que, dix ans après son lancement, la formule a trouvé son public, même s'il reste relativement restreint (50.000 exemplaires vendus en moyenne par numéro ${ }^{2}$ ) par rapport à d'autres types de publications, tels que les newsmagazines.

Si la redécouverte du journalisme narratif dans l'espace francophone se déploie depuis environ une décennie sur papier, on peut remarquer que, très récemment, celui-ci a également pris place sur le web. En effet, au-delà des sites internet des revues proposées en librairies et en kiosques sur lesquels on peut lire certains récits, des pure players qui peuvent être affiliés au journalisme narratif ont éclos sur la toile.

2 Source : https://social.shorthand.com/DlfClemence/ny5JDwM7JY/la-revue-xxiest-elle-revolutionnaire 
Et lorsque l'on évoque ce phénomène, deux sites sont régulièrement cités : Les Jours et Le Quatre Heures. Leur apparition extrêmement récente - ils naissent tous les deux en 2016 - a été largement médiatisée et beaucoup ont salué cette initiative de leurs confrères ; un travail universitaire a même été consacré à leur étude comparée (Joset, 2016). Les journalistes y déploient un « journalisme ultranarratif ${ }^{3}$ » dans le premier et des « reportages long-format, multimédias, et immersifs ${ }^{4}$ » dans le second. Nous retrouvons dans ces deux pure players cette envie de mettre la narration au service de l'expérience et de la compréhension du réel grâce aux outils numériques.

La recherche scientifique envisage régulièrement le journalisme narratif du point de vue de la narratologie, pour analyser les ressorts de la construction du récit (en particulier Pélissier, 2012). Certains auteurs, à l'instar de Jacqueline Marino (2016), commencent à aborder le phénomène de la transposition numérique du journalisme narratif en s'intéressant aux dispositifs techniques utilisés. Cette problématique est pourtant centrale car, comme le démontre très bien Emmanuël Souchier, « il n'y a pas de transformation technologique qui ne soit accompagnée d'une transformation des modes de faire et par-là même des modes de penser » (Souchier, 1996).

Nous proposons ici d'analyser les pratiques des professionnels du journalisme narratif en partant de ce que Jeanneret et Souchier nomment l'énonciation éditoriale. «Cet autre niveau d'énonciation définit les formes même qui rendent les textes possibles, qui lui permettent d'avoir une visibilité, [elle] est ce par quoi le texte peut exister matériellement, socialement, culturellement... aux yeux du lecteur [...]» (Jeanneret \& Souchier, 2005). L'énonciation éditoriale a une incidence majeure sur la construction du récit que font les journalistes. Les « écrits d'écran » ne peuvent se déployer de la même manière que sur un support papier. Ce postulat pris dans le cadre du journalisme narratif suppose que le journaliste déploie des stratégies différentes quant à la mise en place de son récit. Le passage sur le web conditionne la construction des récits du journalisme narratif. En migrant sur le web, les ressources des professionnels sont démultipliées, ils peuvent faire appel à l'hypertexte, au son, à la vidéo, à la photographie, proposer de

3 Le qualificatif est utilisé par les fondateurs - issus pour la plupart du quotidien $L i$ bération - du pure player sans qu'il ne soit réellement défini : https://lesjours.fr/ obsessions/vie-jours/ep1-origine/

4 Qualification des fondateurs : https://lequatreheures.com/presentation/ 
multiples parcours dans le récit... En s'intéressant à ce processus, nous pourrions alors répondre au vœu de Marc Lits qui souhaite une "narratologie refondée » : une " hypernarratologie », qui permettrait, en la croisant avec la «prise en compte des avancées technologiques, des supports nouveaux, des évolutions des usages et des publics [...] », une saisie de «l'homme socialisé comme un animal narrativisé, traversé par des récits construits, selon des formes radicalement nouvelles et ouvertes » (Lits, 2008).

\section{Le numérique : outil d'une expérientialité renouvelée}

L'information est présente partout quasiment en temps réel et gratuitement et, dans un contexte difficile du point de vue économique et extrêmement concurrentiel, les médias se doivent d'être présents en ligne. Outre cette injonction à proposer des contenus en ligne, on note une uniformisation de l'information et de la structure globale des sites sur lesquels elle est publiée. C'est ce que Dominique Maingueneau appelle un "lissage des différents génériques ». Pour lui, désormais, « le principal souci est la mise en scène de la communication, qui mobilise massivement les ressources proprement verbales, multimodales (images fixes, mouvantes et sons) et les opérations hypertextuelles » (Maingueneau, 2017). Si la prédominance de la « scénographie » est devenue une problématique commune aux sites d'information, le journalisme narratif y apporte une dimension supplémentaire. La mise en scène ne constitue pas uniquement une stratégie de différenciation à l'égard des concurrents, elle est réalisée dans un autre dessein. Il s'agit ici d'utiliser les « ressources verbales, multimodales [...] et les opérations hypertextuelles » (Maingueneau, 2017) pour faire ressentir une expérience aux lecteurs.

\subsection{La fin du récit linéaire grâce au rich media}

Comme rappelé auparavant, le journalisme narratif s'appuie sur un « arc narratif » faisant intervenir des protagonistes qui connaissent une complication dans leur vie quotidienne, dont le journaliste fait état en créant une tension narrative, et qui trouve un dénouement à la fin du récit. Dans des revues comme XXI ou Feuilleton, le fil narratif se déroule de manière linéaire ; sur les sites web, et notamment dans les pure players comme Les Jours ou Le Quatre Heures, le lecteur quitte cette linéarité. Les textes côtoient des vidéos, des animations, des liens 
hypertextes, chacun de ces éléments constituant des micro-récits locaux, participant à un " métarécit » dans lequel le lecteur a un rôle central (Barboza, 2006). Ainsi nous retrouvons cette non-linéarité du récit dans les dispositifs mis en ligne dans Les Jours et Le Quatre Heures. Le premier propose par exemple des « épisodes »: les sujets d'actualité - appelés « obsessions 》 - sont déclinés en plusieurs chapitres, ayant un titre distinct, se suivant les uns les autres, tout en étant autonomes. Chaque épisode se suffit à lui-même et peut être consommé et compris indépendamment des autres. Par ailleurs, dans ce pure player créé par des anciens de Libération, comme dans celui des jeunes journalistes du Quatre Heures, le récit est éclaté entre différents modules. Les Jours structurent par exemple leur narration en un long texte, disposé à gauche de l'écran, contenant des liens hypertextes qui renvoient le plus souvent à des ajouts d'informations dans une colonne située à droite. Les hyperliens, qui renvoient traditionnellement à des sources externes, emmènent ici le lecteur vers des développements d'information supportés par le même site, dans une même page-écran ou vers une autre page interne.

Considérant aux côtés d'Alain Joannès que le multimédia est composé de « texte, de la vidéo et des animations électroniques dispersés ", ce qui se donne à voir ici est davantage à analyser en terme de rich media. En effet, la scénographie de l'information y est organisée « en tenant en compte des apports particuliers de chaque mode d'expression, auxquels s'ajoute l'interactivité »(Joannès, 2009). Ainsi, nous ne sommes pas face à des contenus juxtaposés les uns aux autres, mais bien face à une utilisation réfléchie des spécificités des modes d'expression disponibles sur le web en vue de construire un récit dans lequel l'expérientialité joue un rôle majeur. Le tableau 1 explicite ces spécificités d'après le travail d'Alain Joannès. 
Tableau 1. Spécificités des modes d'expression d'après le travail d'Alain Joannès

\begin{tabular}{|c|l|}
\hline Modes d'expression & \multicolumn{1}{c|}{ Spécificités } \\
\hline Textes & $\begin{array}{l}\text { Accessibilité ; précision ; qualification ; nuances ; princi- } \\
\text { paux vecteurs de l'information }\end{array}$ \\
\hline $\begin{array}{c}\text { Sons } \\
\text { (bruit, musique, voix) }\end{array}$ & $\begin{array}{l}\text { Expressivité ; accentuation ; suggestivité ; pouvoir d'évo- } \\
\text { d'informations si reliés à des images mentales chez le } \\
\text { récepteur ; relèvent de la dénotation pour les bruits, de } \\
\text { la dénotation pour la musique, ou d'informations et de } \\
\text { dénotation pour la voix }\end{array}$ \\
\hline $\begin{array}{c}\text { Images fixes } \\
\text { phie, carte, graphique) }\end{array}$ & $\begin{array}{l}\text { Référence à une réalité ; densité d'informations ; traduc- } \\
\text { tion des abstractions ; concentration de très nombreuses } \\
\text { données ; véhiculent à la fois des informations explicites } \\
\text { et implicites, des sensations et des émotions }\end{array}$ \\
\hline $\begin{array}{c}\text { Images animées } \\
\text { (vidéo, animation } \\
\text { électronique) }\end{array}$ & $\begin{array}{l}\text { Mouvement ; expressivité ; argumentation ; remplace- } \\
\text { ment possible des autres modes d'expression en concen- } \\
\text { trant leurs apports ; faciliter la compréhension de réalités } \\
\text { peu accessibles aux mots }\end{array}$ \\
\hline Liens & $\begin{array}{l}\text { Profondeur ; élargissement ; modularité ; création d'une } \\
\text { nouvelle manière d'assimiler les contenus ; relations } \\
\text { dynamiques entre objets ayant du sens ou entre objets / } \\
\text { internautes }\end{array}$ \\
\hline
\end{tabular}

« L'originalité de l'approche rich media dépend moins du nombre et de la diversité des modes d'expression mobilisés pour un reportage que de la manière de répartir ces modes d'expression » (Joannès, 2009). Au sein de nos deux pure players, les modes d'expression utilisés sont relativement similaires. Les récits se structurent autour de textes, de liens hypertextes, d'images fixes (essentiellement de la photographie) et d'images animées (notamment la vidéo, qui fait toujours office d'introduction dans Le Quatre Heures). Il faut cependant souligner que, chez Les Jours, les médias sont plus diversifiés, puisqu'un grand nombre 
d' « obsessions » est associé à une playlist ${ }^{5}$, l'équipe réalise également des émissions en direct $^{6}$ et propose des quiz ${ }^{7}$.

Néanmoins, dans un cas comme dans l'autre, la distribution des modes d'expression varie selon les sujets, ce qui fait écho à la répartition réfléchie évoquée par Joannès. L'exemple d'un des récits publiés dans Le Quatre Heures en est significatif : « Casse moyenne » (Jeanticou \& Paillard, 2017) donne à voir la situation des villes moyennes françaises, grâce au parcours des journalistes dans trois villes, Vierzon, Nevers, Roanne. Ces derniers s'attachent à décrire les combats de trois protagonistes pour sauver leurs villes. Ici, les textes, les vidéos, les photographies, les sons, les liens hypertextes se répondent majoritairement les uns aux autres. Les journalistes semblent avoir pris en compte les spécificités de chaque médium pour donner vie à leur récit et faire vivre aux lecteurs une expérience de cette réalité. Ainsi, l'article se compose de trois ensembles distincts consacrés à un lieu et un protagoniste, donnant finalement une vue globale des différentes difficultés rencontrées par ce type de villes. « Casse moyenne » s'ouvre sur ce que l'on pourrait appeler une vidéo d'ambiance (figure 1) : une caméra fixe filme un défilé de pom-pom girls dans une ville où l'on aperçoit des immeubles défraîchis et des commerces fermés, les spectateurs étant quant à eux peu nombreux. Aucune voix off ne se fait entendre dans cette vidéo, les bruits (bribes de conversation des passants, musique du défilé) sont conservés en l'état.

D’emblée, le lecteur est plongé au cœur du récit, à l'instar d'un observateur sur place, il est emmené, grâce à cette vidéo d'ambiance, dans une scène qui, comme l'explique Joannès, " donne une idée de phénomènes complexes ou abstraits » (2009) décrits dans l'ensemble de l'article. Chaque ensemble est introduit de la même manière. Cet usage de la vidéo vise à donner du réalisme aux mots du texte, mais aussi une certaine intensité dans la mesure où les images visent à créer une sorte d'immersion instantanée dans une atmosphère particulière.

5 Par exemple, pour l'obsession « La cause du people », la playlist se compose des chansons suivantes : « Les amours des journaux » d'Adamo ; "Paparazzi » de Philippe Catherine ; «Paparazzi » de The Baseballs; « Piece of Me » de Britney Spears ; « The Way I Am » d'Eminem et « Scandal » de Queen.

6 Par exemple : https://lesjours.fr/obsessions/drahi-sfr/ep19-live/

7 Par exemple : https://lesjours.fr/obsessions/pole-financier/ep38-quiz-magouillotron/ 


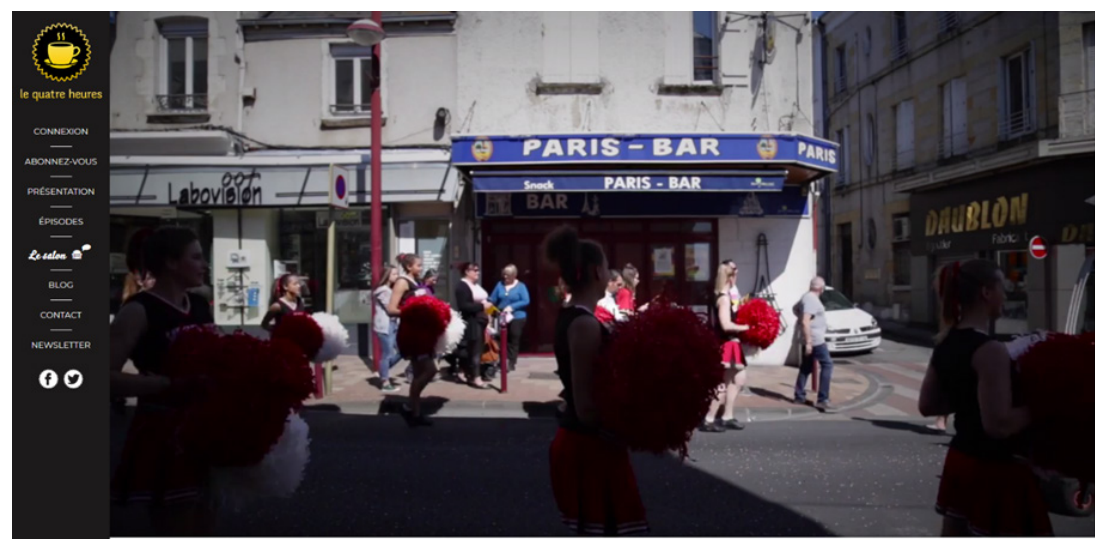

Figure 1. Capture d'écran Le Quatre Heures, « Casse moyenne » : vidéo introductive

Les textes rédigés par les journalistes sont essentiellement composés de citations, de récits de vie et de descriptions des protagonistes du récit. Des données d'ensemble, telles que des statistiques, sont également proposées. Là aussi, nous retrouvons ce que Joannès pointe : le texte est un vecteur central d'informations et permet la qualification, notamment des personnages dont on relate la vie intérieure. Ces descriptions peuvent constituer autant de points d'ancrage pour une projection de l'internaute dans l'expérience du reportage et de ses acteurs.

Le récit est également ponctué de photographies, dont beaucoup sont des portraits des protagonistes de l'histoire, les personnes mais aussi les bâtiments (commerces vides, usine à l'abandon, monument patrimonial). Les photographies de personnes sont susceptibles de générer de l'empathie chez le lecteur qui peut mettre des visages sur des noms, comme avec ce vieil homme seul dans un hôtel vide, le regard au loin et la mine triste (figure 2).

Il est intéressant de noter que ces photographies sont publiées sans légende et, si la plupart d'entre elles sont explicitées dans le texte, bien qu'aucun lien ne soit formulé clairement entre les deux, certaines ne font l'objet d'aucune explication. Le lecteur est laissé seul face à ces photographies, ce qui semble devoir le pousser à un travail de lecture et d'interprétation personnel. L'absence de guide quant à la lecture de ces photographies engage le lecteur à faire appel à ses cadres d'interprétation et à construire sa propre signification des clichés qui lui sont donnés à voir. 

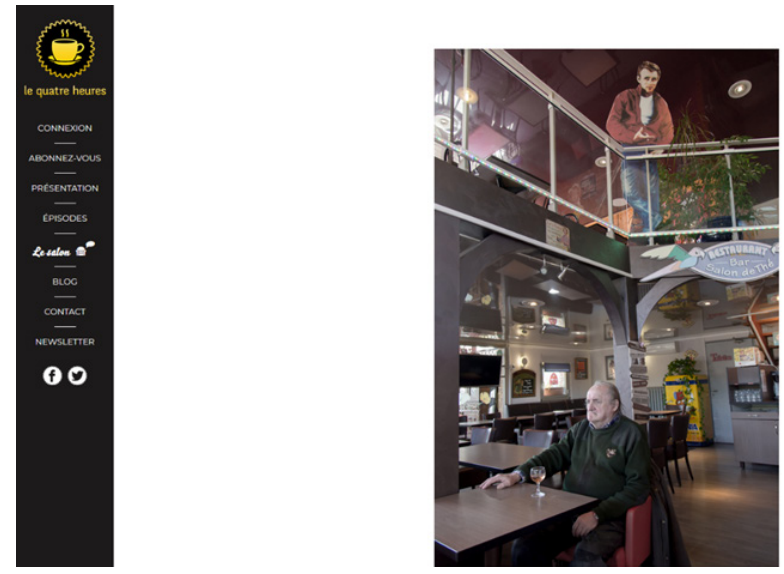

Figure 2. Capture d'écran Le Quatre Heures, « Casse moyenne » : vieil homme seul dans l'hôtel

Par deux fois, les journalistes utilisent des bandes sons qui donnent à entendre la voix des protagonistes principaux pour les villes de Vierzon et Roanne. Rémy et Sophie, qui se battent chacun à leur manière pour que leurs villes ne meurent pas un peu plus, se livrent dans ces formats audio. Le premier confie sa réaction face au refus du maire de Vierzon de faire de l'usine historique, désormais fermée, un musée ; la seconde se remémore ses souvenirs d'adolescente lors de la destruction d'une usine importante de Roanne. Ces témoignages audio font entendre l'émotion dans la voix de ces personnages centraux : la colère chez l'un, la tristesse et la résignation chez l'autre. Une nouvelle fois, le format choisi constitue un mode d'expression particulièrement apte à transmettre ces sentiments, au-delà des mots.

À l'instar des descriptions psychiques et des représentations photographiques des personnages, ces enregistrements sonores constituent des supports d'incarnation (embodiment) de l'expérience, définie comme centrale dans l'expérientialité par Fludernik (1996).

Enfin, chose peu commune dans le monde des sites d'information, un seul lien hypertexte pointant vers une ressource externe est proposé dans cet article. Il constitue un des éléments centraux de la chute du premier ensemble consacré à Vierzon. La fin de cet ensemble, comme les autres par ailleurs, s'achève sur une note d'optimisme, ici les projets de Rémy pour faire revivre sa ville. Parmi ces projets, il explique vouloir « organiser des visites guidées autour de Jacques Brel, qui chantait en 1968 “T'as voulu voir Vierzon, et on a vu Vierzon”. Afin que d'autres, 
quarante ans plus tard, nourrissent la même envie. » Les paroles de Brel constituent le seul lien hypertexte vers une ressource extérieure au site : une prestation scénique de la chanson «Vesoul » de Jacques Brel.

\subsection{La place du "lect-acteur"}

Un autre élément attire l'attention lorsque l'on s'intéresse au journalisme narratif sur le web : la place du lecteur, devenu un « lectacteur » selon les termes de Weissberg (1999). Cette place a largement été modifiée dans le journalisme traditionnel et bon nombre de chercheurs se sont intéressés à ce phénomène d'interactivité. Comme le signale Julia Bonaccorsi,

La relation entre le sens et la forme est profondément modifiée et par là, le pouvoir du lecteur dans le processus de signification : le texte à l'écran est doublement «produit » dans la lecture, par son affichage technologique (paramètres logiciels et support technique) et par la participation du lecteur qui doit agir sur le dispositif technique par le biais d'autres signes. (Bonaccorsi, 2017)

Dans le cadre du journalisme narratif, l'interactivité permise par le web donne un pouvoir au lecteur, il peut choisir, ou non, de regarder les vidéos, d'écouter les voix, de lire l'ensemble du récit ou seulement quelques épisodes... Cette interactivité lui confère également un degré d'engagement supplémentaire dans le récit. Le lecteur crée son propre parcours au sein des micro-récits mis en scène par le journaliste. Ainsi, la narration est double : le journaliste organise une histoire, tandis que le lecteur crée la sienne à partir de la première (Lits, 2012 ; Amato, 2015). Ce qui est particulièrement intéressant dans ce cadre réside dans le fait que " cette "auto-narration" [...] serait à considérer comme une possible source de réflexivité amplifiant le projet documentaire. Elle irait dans le sens d'une implication plus profonde, car ancrée dans la décision » (Amato, 2015). En faisant le choix de regarder telle vidéo ou de visionner tel diaporama, on peut penser qu'un rapport particulier à l'expérientialité se met en place dans la mesure où ces visionnages résultent d'une décision personnelle qui nécessite une implication du lecteur. Celui-ci choisit de s'orienter vers cette proposition de microrécit car le chemin qui lui permet d'y accéder trouve une résonance dans son expérience personnelle. 
Cette modularité du récit induit une forme nouvelle d'engagement du lecteur quant à la création du sens du récit. Il est « actif [...] dans l'attribution d'une signification aux événements » (Joannès, 2007). En effet, au sein du journalisme narratif qui, grâce au rich media, donne à dessein des formes d'expression différentes de l'expérience personnelle des protagonistes, l'expérientialité est permise par le fait que le lecteur doit s'emparer des différents modules, dans leur totalité ou en partie, les lier entre eux afin de faire sens. Ainsi ce caractère multimédia permet aux lecteurs de construire activement des significations quant aux expériences de vie qui lui sont exposées.

Dans ce type de journalisme narratif, l'expérientialité particulière construite par le rich media pourrait ainsi être au service de la compréhension du phénomène décrit, puisque celle-ci permet une forme de projection du lecteur en son sein. C'est ainsi que l'on peut envisager les différents renvois proposés dans le texte principal de l'épisode 1 de « l'obsession » consacrée aux cheminots chez Les Jours (Eychenne \& Castanier, 2018). Il s'agira, par exemple, en cliquant sur le nom des protagonistes qui interviennent dans le récit, de découvrir d'où ils parlent et par corrélation de décrire de manière précise leur expérience passée mais aussi actuelle : qui sont-ils ? Quelle est leur histoire personnelle ? Quel est leur lien avec la SNCF ? Quelle est leur position quant à la réforme proposée par le gouvernement? Pour chacun des quatre protagonistes s'exprimant dans cet épisode, un portrait est en effet dressé dans lequel est décrit son parcours, notamment au sein de la SNCF (figure 3).

Ces portraits manifestent pleinement la présence des protagonistes du récit, qui sont de plus représentés en photographie, et permettent aux lecteurs de s'approprier leurs expériences. Ainsi, ces portraits sont autant d'éléments, parmi d'autres, qui contribuent à conférer au récit un sens, mais c'est au lecteur d'aller chercher, de construire ce sens.

La construction du récit et les techniques de narration sont donc complétées par l'utilisation du rich media qui confère au lecteur une nouvelle place, qui chemine dans la mise en scène proposée par le journaliste. On voit alors se mettre en place une forme de personnalisation de l'expérience en fonction des choix effectués par le lecteur. 


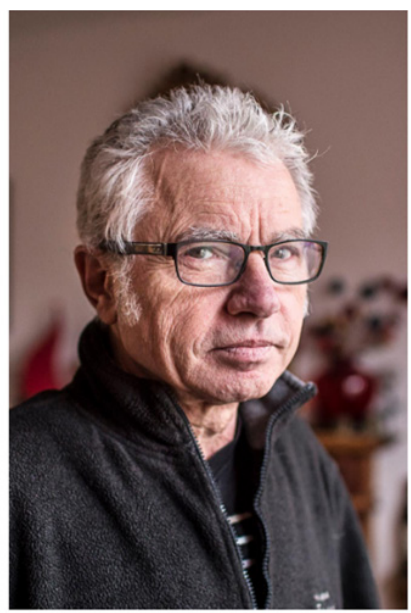

- CHEMINOTS

$\times$

Dominique Canu

Retraité de la SNCF, 62 ans

Entrée à la SNCF en 1974 avec un BEP d'électromécanicien, Dominique Canu a suivi deux ans de formation pour pouvoir travailler à la signalisation. Un poste sensible : à l'époque, sa ligne, entre Aulnay-sous-Bois et Bondy, était traversée par une dizaine de passages à niveau. Pendant 28 ans, il a assuré des services d'astreinte, réveillé en pleine nuit par les aiguilleurs pour intervenir sur les voies après sa journée de travail. Nomade syndical, il est passé par la CGT, la CFDT, puis SUD Rail. En retraite depuis 2014, il préside l'amicale les locataires de la cité cheminote de Chelles et Brou-surChantereine, en Seine-et-Marne, où il vit toujours.

Photo Yann Castanier pour Les Jours

Figure 3. Capture d'écran Les Jours, «Les cheminots », épisode 1 : portrait d'un protagoniste

\section{Du storytelling au « storyliving »}

Parallèlement au développement des pure players tels que Les Jours ou Le Quatre heures, et sous l'impulsion des recherches sur le marketing dit expérientiel ou immersif, la réalité virtuelle, la réalité augmentée, les vidéos à $360^{\circ}$ ou encore les hologrammes tendent à devenir de nouveaux outils pour les journalistes. Si en marketing l'objectif est de renforcer la relation entre le client ou le prospect et la marque, pris dans le cadre du journalisme, le recours à ces derniers est à entendre comme des ressources supplémentaires quant à l'expérientialité du récit. Grâce à ces nouvelles technologies, l'immersion n'est plus seulement à entendre comme une « opération cognitive de projection » (Oléron, 2015) mais comme un engagement physique dans un univers virtuel. Pris dans le cadre du journalisme, c'est ce que Frank Rose nomme le deep media pour mettre l'accent sur cette immersion recherchée (Rose, 2011). Il explique dans un entretien avec Henry Jenkins : « Deep media puts the focus on the goal: To enable members of the audience $[. .$.$] to delve into a story at any level of depth they like,$ to immerse themselves in it » (Jenkins, 2011). Du rich media nous passons donc au deep media. 


\subsection{La gamification du journalisme}

Cette recherche de l'immersion du public a d'abord pris corps dans ce que l'on appelle la gamification (ou ludification) de l'information. Largement étudiée dans le domaine de la formation, la gamification vise à mettre à profit les techniques développées dans les jeux vidéo pour créer notamment des serious games ou des newsgames. Certains sont créés par des journalistes en tant que nouveaux supports de transmission de l'information. À l'instar de jeux vidéo classiques, l'acteur principal est le joueur, c'est lui qui agit sur et dans l'histoire. Ainsi, dans ces récits, le lecteur devenu acteur est face à un autre type d'expérientialité où ses propres choix peuvent influencer l'expérience des protagonistes au sein de l'histoire.

Les premiers avatars de cette gamification naissent au début des années 2000 avec les webdocumentaires. Récompensé par un World Press Photo dans la catégorie «multimédia non linéaire » en 2011, le webdocumentaire Prison Valley (Dufresne \& Brault, 2010) constitue l'un des exemples les plus aboutis et les plus commentés du deep media en la matière. Dans celui-ci, le joueur commence par prendre connaissance des lieux et du contexte par une vidéo introductive complétée de voix off : « l'industrie de la prison » de Cañon City dans le Colorado. Après s'être enregistré dans l'un des motels de la ville (figure 4), le joueur se retrouve dans une chambre où plusieurs options s'offrent à lui (figure 5).

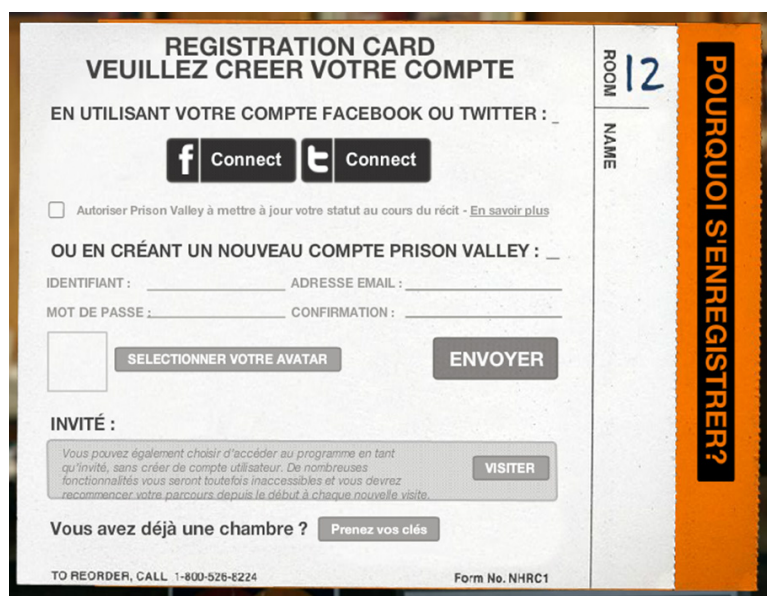

Figure 4. Capture d'écran, Arte, Prison Valley : enregistrement au motel 


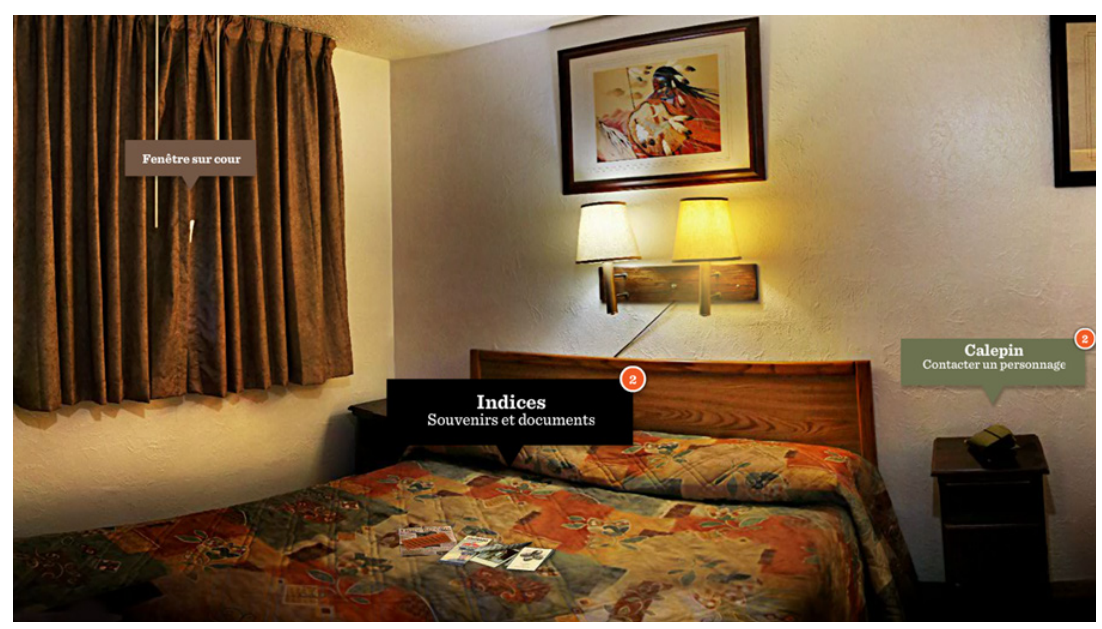

Figure 5. Capture d'écran, Arte, Prison Valley : chambre du motel

Chacune de ces actions le mène vers des contenus différents, textes, photographies, vidéos, carte géographique, liens hypertextes externes et internes, interactions virtuelles avec des protagonistes. Ces choix lui permettent de créer son propre parcours. Ils ont été pensés et organisés par les journalistes mais c'est bien le joueur qui est actif dans ce webdocumentaire, voire qui prend la place des professionnels puisqu'il doit recueillir des indices dispersés dans l'univers virtuel et leur donner un sens. Nous sommes ici dans une expérientialité qui prend appui sur un récit modulaire poussé à son paroxysme, la voix des journalistes est quasiment absente et la liberté laissée au joueur est remarquable, même si elle reste limitée par le fait qu'il évolue toujours dans des scénarios préétablis par le(s) auteur(s). Le public est donc au cœur du dispositif. Les sites web de médias comme France Télévisions, Arte ou Le Monde ont désormais des rubriques dédiées à ce type de projets, signe que le renouvellement des techniques de narration par le numérique est une tendance prise au sérieux par les professionnels.

\subsection{La réalité virtuelle au service de l'expérientialité}

Une autre tendance est également à distinguer. Celle-ci s'appuie sur des technologies récentes et place le public dans une situation d'engagement physique avec l'univers narratif. Ce que l'on appelle le journalisme expérientiel ou immersif repose en effet sur la réalité virtuelle. 
Prolongement de la gamification de l'information, certains médias proposent ainsi à leur public des narrations inédites.

La réalité virtuelle (VR), qui fait l'objet de recherches scientifiques dès la fin des années 1950 bien que l'expression virtual reality n'apparaisse que dans les années 1980, s'est énormément développée ces dernières années. Celle-ci a pour finalité, selon Philippe Fuchs, de « permettre à une personne (ou à plusieurs) une activité sensi-motrice dans un monde artificiel, qui est soit imaginaire, soit symbolique, soit une simulation de certains aspects du monde réel ». L'auteur insiste et explicite cette notion d'activité sensi-motrice : « [Elle est] employé[e] pour signifier qu'au fondement de la réalité virtuelle la personne perçoit et agit physiquement. Bien évidemment, il en découle que la personne a aussi une activité cognitive " (Fuchs, 2003). Par le biais de casques, mais aussi de cabines ou de smartphones, cette technologie est particulièrement intéressante pour faire vivre au public un récit. Celui-ci ne passe plus uniquement par des mots, par des techniques de narration, ou par l'usage du rich media, il s'appuie aussi sur un engagement physique du récepteur dans un monde virtuel.

Cette simulation du monde réel s'appuie sur de longues heures de tournage, de prises de vue et de sons sur place, qui seront ensuite assemblés et recomposés par des journalistes et des web designers. Une des figures de proue du phénomène est Nonny de la Peña qui se lance très tôt dans le documentaire immersif via la réalité virtuelle, notamment avec son documentaire immersif Project Syria (de la Peña, 2014) dans lequel elle place le spectateur - équipé de son casque de VR - au cœur des bombardements dans les rues d'Alep en Syrie. Elle explique à $L^{\prime} O b s$ :

Je pense que c'est un medium qui peut enlever le filtre du journalisme, et laisser les gens être les témoins de leur propre histoire. [...] Ça crée une empathie qui dépasse de loin tous les autres types de médias que nous avons actuellement pour raconter ce genre d'histoires. Car on ne voit pas le monde à travers nos yeux, mais notre corps entier. (Richard, 2014)

La « Godmother » du journalisme immersif, comme elle est surnommée, a fait des émules et de grands médias, en particulier américains, se sont engagés dans la VR. Tel est le cas du New York Times, qui a lancé son application NYT VR et offert à ses abonnés des Google 
Cardboard, de la chaîne CNN, qui a lancé une plateforme CNN VR, ou encore d'Al Jazeera, du Huffington Post, du Guardian, etc. En France, la tendance commence à se développer, par exemple Le Monde et Le Parisien relaient régulièrement des reportages en VR ; notons enfin la création d'un pure player français, Targo, qui y est entièrement dédié.

Dans chacune de ces expériences le storytelling semble laisser place au «storyliving ». Nous sommes au-delà de la volonté de recréation des sentiments de personnes impactées par des faits d'actualité et le lecteur est censé s'identifier pleinement à ces personnes et à leurs sentiments, le but est de faire ressentir véritablement au public la situation exposée ${ }^{8}$.

\section{Conclusion}

Ces technologies seraient-elles l'avenir du journalisme, et plus particulièrement du journalisme narratif ? La narration journalistique devra-t-elle nécessairement prendre appui sur celles-ci, collant ainsi à un environnement toujours plus technologique ? Il est impossible de répondre formellement à cette question. Cependant, ce n'est sans doute pas un hasard si Google, par l'intermédiaire de sa Digital News Initiative dotée de 150 millions d'euros, se propose d'encourager les projets journalistiques européens s'appuyant sur ces nouvelles technologies. Il faudra néanmoins probablement beaucoup de temps pour que ces technologies s'imposent réellement. Leurs coûts restent extrêmement élevés, notamment pour des médias fragilisés économiquement, ce qui explique qu'elles restent encore réservées à certains grands groupes. De plus, dans le cadre de la VR, les casques sont encore peu répandus dans la population.

Enfin, la pratique soulève des questions quant à la véritable compréhension des questions d'actualité. Lorsqu'un joueur enquête dans Prison Valley, est-il poussé à avancer par l'envie de récolter des indices - et dans une certaine mesure de « gagner » - ou a-t-il réellement la volonté de comprendre cette industrie carcérale ? Comment s'assurer qu'il ait récolté les indices suffisants pour avoir en main l'ensemble des tenants et des aboutissants du récit ? Dans le cadre de la VR, la simulation des émotions ne se fait-elle pas au détriment de la compréhension? Valérie Croissant l'explique très bien au Monde : «D'un point de vue

8 À cet égard, voir l'article d'Angelina Toursel et Philippe Useille dans ce même numéro de Recherches en Communication. 
scientifique et intellectuel, on considère que c'est plutôt la prise de distance qui permet l'intelligibilité » (Truong, 2016). Si l'on peut considérer que la dimension expérientielle du journalisme narratif enrichit la compréhension des faits d'actualité, il ne faudrait cependant pas que le récit s'y réduise. Cela pourrait alors se faire aux dépens de la connaissance : immersion et compréhension doivent se compléter. Une chose reste certaine, le recours à ces nouvelles technologies est le symbole d'un journalisme qui cherche à se réinventer.

\section{Références}

Amato, E. A. (2015). Le webdocumentaire et ses ressorts fonctionnels, au croisement du film documentaire et de la narration hypermédia. Dans G. Soulez \& K. Kitsopanidou (Éds), Le Levain des médias. Forme, format et média (pp. 203-212). Paris : L'Harmattan.

Barboza, P. (2006). Fiction interactive, «métarécit» et unités intégratives. Dans P. Barboza \& J.-L. Weissberg (Éds), L’Image actée. Scénarisations numériques, parcours du séminaire « L'action sur l'image » (pp. 99-121). Paris : L'Harmattan.

Bonaccorsi, J. (2017). Approches sémiologiques du web. Dans C. Barats (Éd.), Manuel d'analyse du web en Sciences Humaines et Sociales (pp. 125-146). Paris : Armand Colin.

Caracciolo, M. (2013). Experientiality. Dans P. Hühn, J. Pier, W. Schmid \& J. Schönert (Éds), The Living Handbook of Narratology. Hamburg: Hamburg University. Disponible à : http://www.lhn.uni-hamburg.de/node/102.html

de la Peña, N. (2014). Project Syria. Disponible à : http://emblematicgroup.com/experiences/project-syria/

Dufresne, D. \& Brault, P. (2010). Prison Valley - un webdocumentaire sur l'industrie de la prison. Arte. Disponible à : http://prisonvalley.arte.tv/?lang=fr

Eychenne, A. \& Castanier, Y. (2018). Cheminots. Les Jours. Disponible à : https:// lesjours.fr/obsessions/sncf-cheminots/ep1-cite-cheminote/

Fuchs, P. (2003). Le Traité de la réalité virtuelle, volume 1 : Fondements et interfaces comportementales ( $2^{\mathrm{e}}$ éd.). Paris : Presses de l'École des Mines.

Fludernik, M. (1996). Towards a « Natural » Narratology. London : Routledge.

Jeanneret, Y. \& Souchier, E. (2005). L'énonciation éditoriale dans les écrits d'écran. Communication \& Langages 145(1), 3-15.

Jeanticou, R. \& Paillard, R. (2017). Casse moyenne. Le Quatre Heures. Disponible à : https://lequatreheures.com/episodes/casse-moyenne/

Jenkins, H. (2011). « Deep Media, » Transmedia, What's the Difference?: An Interview with Frank Rose (Part One) ». Disponible à : http://henryjenkins.org/blog/2011/01/ deep_media_transmedia_whats_th.html

Joannès, A. (2007). Le Journalismè à l'ère électronique. Paris : Vuibert.

Joannès, A. (2009). Communiquer en rich media: Structurer les contenus en optimisant textes, sons, images et liens ( $1^{\text {re }}$ éd.). Paris : CFPJ Éditions.

Joset, E. (2016). Slow media : émergence d'un journalisme narratif sur le web. Étude comparative du dispositif mis en place par les pure players Le Quatre Heures et 
Les Jours (Mémoire de fin d'études, Master web éditorial). Université de Poitiers. Disponible à : http://www.elena-joset.fr/memoire.pdf

Lallemand, A. (2011). Journalisme narratif en pratique. Bruxelles : De Boeck.

Lits, M. (2007). L'information à l'heure médiatique numérique ou la fin du récit médiatique ? Recherches en Communication 28, 81-89.

Lits, M. (2008). Du récit au récit médiatique. Bruxelles : De Boeck.

Lits, M. (2012). Storytelling, réévaluation d'un succès éditorial. Dans N. Pélissier \& M. Marti (Éds), Le Storytelling. Succès des histoires, histoire d'un succès (pp. 23-38). Paris : L'Harmattan.

Maingueneau, D. (2017). Genres de discours et web : existe-t-il des genres web ? Dans C. Barats (Éds.), Manuel d'analyse du web en Sciences Humaines et Sociales (pp. 74-98). Paris : Armand Colin.

Marino, J. (2016). Reading Screens: What Eye Tracking Tells Us about the Writing in Digital Longform Journalism. Literary Journalism Studies 8(2), 139-149.

Oléron, A. (2015). La méta-herméneutique, ou l'analyse des pistes interprétatives. Acta Fabula 16(1). Disponible à : http://www.fabula.org/acta/document9099.php

Pélissier, N. (2012). Le journalisme narratif : vecteur privilégié du storytelling ou antidote à ses dérives? Dans N. Pélissier \& M. Marti (Éds), Le Storytelling. Succès des histoires, histoire d'un succès (pp. 117-134). Paris : L'Harmattan.

Pélissier, N. \& Eyries, A. (2016). Le développement des compétences narratives, un nouveau défi pour la formation au journalisme? Dans A. Kiyindou, F. Barbey \& L. Corroye (Éds), De l'éducation aux médias à l'éducation par les médias (pp. 232-245). Paris : L’Harmattan.

Richard, C. (2014, 29 septembre). La Syrie comme si vous y étiez : faire du journalisme avec la réalité virtuelle. nouvelobs.com. Disponible à : https://www.nouvelobs.com/ rue89/rue89-rue89-culture/20140929.RUE5978/la-syrie-comme-si-vous-y-etiezfaire-du-journalisme-avec-la-realite-virtuelle.html

Rose, F. (2011) The Art of Immersion. How the Digital Generation Is Remaking Hollywood, Madison Avenue, and the Way We Tell Stories. New York: W. W. Norton \& Co.

Souchier, E. (1996). L'écrit d'écran, pratiques d'écriture \& informatique. Communication \& Langages 107(1), 105-119. doi : https://doi.org/10.3406/colan.1996.2662

Truong, A. H. (2016, 3 novembre). Actu immersive : s'émouvoir plutôt que comprendre? Disponible à : https://www.lemonde.fr/idees/article/2016/11/03/actu-immersive-s-emouvoir-plutot-que-comprendre_5024921_3232.html

Vanoost, M. (2013). Journalisme narratif : proposition de définition, entre narratologie et éthique. Les Cahiers du journalisme 25, 140-161.

Weissberg, J.-L. (1999). Présences à distance. Paris : L’Harmattan.

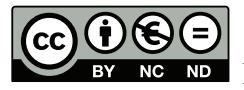

«Attibution - pas d'utilisation Commerciale - Pas de Modification 4.0. International» (CC BY-NC-ND) 